"Risk premiums in life insurance"

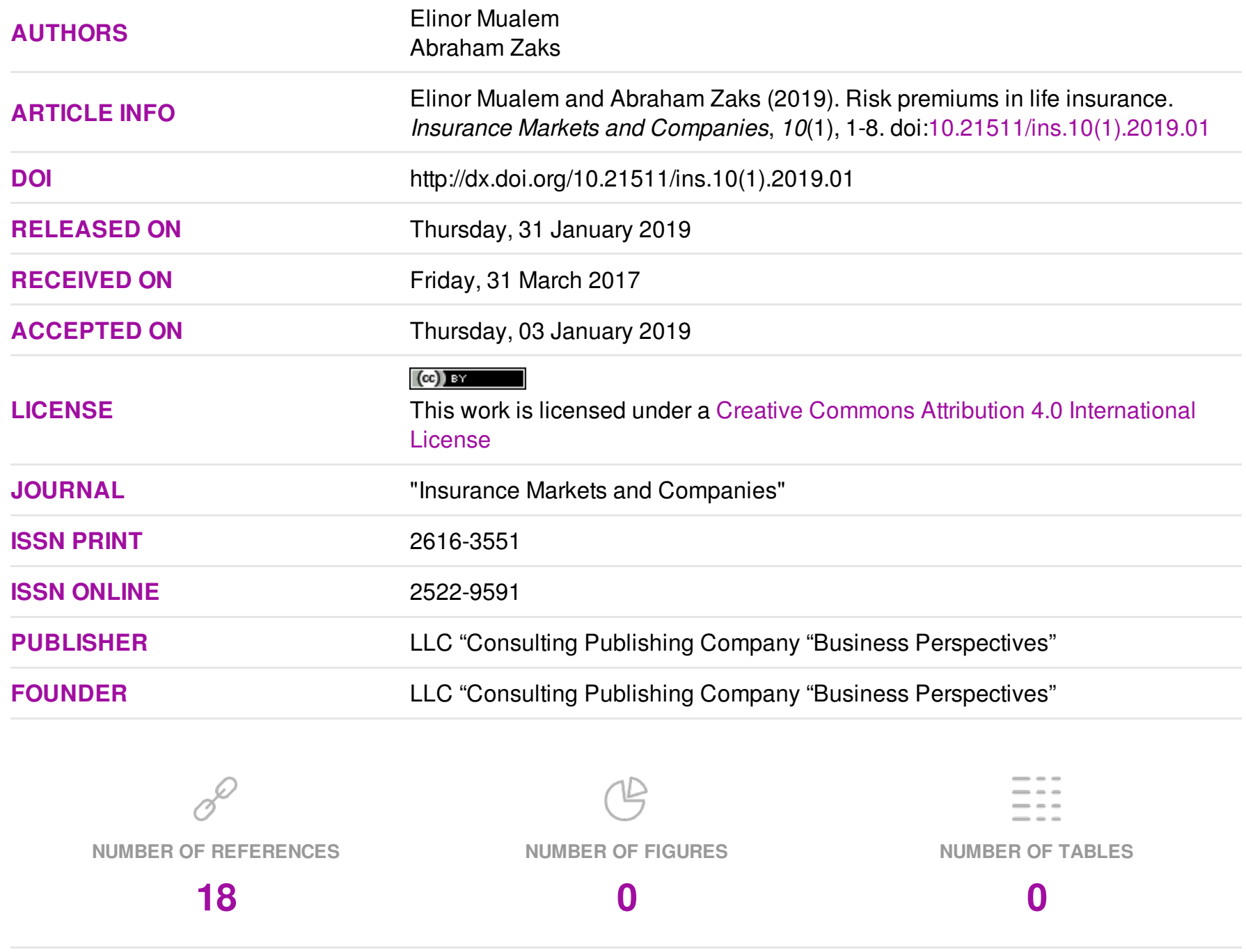

(C) The author(s) 2022. This publication is an open access article. 


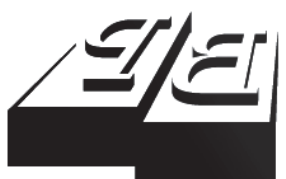

BUSINESS PERSPECTIVES

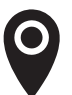

LLC "CPC "Business Perspectives" Hryhorii Skovoroda lane, 10, Sumy, 40022, Ukraine

www.businessperspectives.org

Received on: $31^{\text {st }}$ of March 2017 Accepted on: $3^{\text {rd }}$ of January 2019

(C) Elinor Mualem,

Abraham Zaks, 2019

Elinor Mualem, Department of Mathematics Technion, Haifa, Israel.

Abraham Zaks, Professor Emeritus, Department of Mathematics Technion, Actuarial Research Center, Haifa University, Israel.

\section{RISK PREMIUMS IN LIFE INSURANCE}

\section{Abstract}

For a person insured under an insurance plan the present value of the cash flow of a premium of 1 is a random variable $X$, with expected value $E(X)$ and variance $\operatorname{Var}(X)$, and the present value of the cash flow of a liability of 1 is a random variable $Y$, with expected value $E(Y)$ and variance $\operatorname{Var}(Y)$. The authors follow the classical use of the equation $P E(X)=L E(Y)$ to derive the net premium $P$ for the insurance of $L$. The net premium $p$ for an insurance of 1 is $p=P / L$.

To evaluate the risk of the plan, the authors derive the random variables $\tilde{X}, \tilde{Y}$ from $X, Y$ by a change due to the standard deviations $\sigma_{x}\left(\sigma_{y}\right)$. The random variables $\tilde{X}$, $\tilde{Y}$ are the present values, considering risk of the cash flow of a premium of 1 and of a liability of 1 and the gross premium $\tilde{P}$ is derived from the equation $\tilde{P} E(\tilde{X})=L E(\tilde{Y})$. The gross premium $\tilde{p}$ for an insurance of 1 is $\tilde{p}=\tilde{P} / L$.

There appears a risk load $\delta$, and the gross premium $\tilde{p}$ per unit insured is $\tilde{p}=p+\delta$. For the relative risk load $\delta / p$ the following holds: $\delta / p=(\tilde{p}-p) / p=(\tilde{P}-P) / P$. More on premium and risk may be found in Gerber (1997) and Kaas et al. (2008).

Assuming that $n$ persons are insured in the insurance plan, then the estimates for the risk are reduced to $\sigma_{x} / \sqrt{n}, \sigma_{y} / \sqrt{n}$, where $\left(\sigma_{x} / \sqrt{n}\right)^{2}=\operatorname{Var}(X) / n$, $\left(\sigma_{y} / \sqrt{n}\right)^{2}=\operatorname{Var}(Y) / n$, respectively.

Thus, the risk load $\delta$ decreases when $n$ increases, and there appears a profit in the case of merging insurance portfolios. This profit is due to the reduced risk load and points out the advantage of enlarging $n$ whenever possible, as in the case of using reinsurance.

The standard deviation is relatively simple to calculate, and turns out to be easily used to determine the risk load on the net premium.

Mualem and Zaks (2018) discussed discussed ways of splitting the emerging profit due to a reduced risk load when merging several portfolios.

The authors investigate the emerging profit in the cases of the whole life insurance, the $n$-year endowment insurance, and the pension scheme, and suggest in the conclusion several similar cases in which the methods that are discussed may be used.

\section{Keywords} premium, risk, standard deviation, value and risk of an insurance portfolio

JEL Classification C57, C71, G22

\section{INTRODUCTION}

The authors investigate the emerging profit in the cases of the whole life insurance, the $n$-year endowment insurance, and the pension scheme.

To determine the premium for an insurance plan, the insurance company considers various factors, like the size of the insured group, statistical data adjusted to the insured group, past experience of profit and loss of the insured group. Following the method of the classical insurance theory, the gross premium for the insurance plan includes a risk load, say $\delta=3 \sigma / \sqrt{n}$, so that the gross premium $\tilde{p}$ is $\tilde{p}=p(1+\delta)$, is where $n$ is the number of insured members in the specific insurance plan, $p$ is the net premium for a liability of 1 , and $\sigma$ is the standard deviation. The standard deviation of a random variable may be 
derived from its variance (var), where var $=\sigma^{2}$. The authors study some numeric estimates for the varince (var) and for the standard deviation $\sigma$ for some insurance plans from which there appears an estimate for the relative risk load on the net premium for various insurance plans. Mualem and Zaks (2017) suggest how to manage the reduction in the financial risk that arises when merging portfolios of pension funds, or of mortgage loans, or when some groups that share a similar risk decide to manage jointly their portfolios, and suggest how to evaluate the emerging benefit when the groups are managed jointly, and suggest some ways how to split the emerging benefit due to the reduced risk load among the merging groups.

\section{BASIC INSURANCE EQUATION}

The basic insurance equation is:

$$
p a=A,
$$

where $p$ is the premium paid each period (say yearly) by a member to the insurance company, $A$ is the present value of one unit of benefit at death to a member each period (say yearly) and $a$ is the present value of the payments of a premium of one unit each period (say yearly).

Equation (1) expresses a series of payments of the inssured member to the insurance company, each of $p$ units, as long as the member is alive, that equals the payment of one unit by the insurance company to an insured member in case of his death. The different variables of equation (1) depend on the age of the member, the interest and on the expectation of life. These variables introduce risk to the company, which should be reflected in the premium. However, estimating the actual risk or variance is a non-trivial task. In this paper, the authors investigate different means to estimate the risk. The authors focus on few specific insurance types. Three subtypes of insurances will concern us:

1. Whole life insurance in section 2 .

2. $n$-year endowment insurance in section 3 .

3. Pension scheme in section 4 .

In each of these cases, the authors derive estimates to the risk. The estimates are given using elements, which can be calculated using standard mortality tables, such as the variance of $v^{T_{x}}$ and so forth.

\section{WHOLE LIFE INSURANCE}

In case of the continuous whole life insurance of 1 , the basic equation is:

$$
p \bar{a}_{x}=\bar{A}_{x},
$$

where $\bar{a}_{x}$ is the present value of the net premiums paid by the member, $\bar{A}_{x}$ is the present value of the liability of 1 paid in case of death, $p$ is the yearly premium paid continuously.

In case of the continuous whole life insurance of $L$, the basic equation is:

$$
P \bar{a}_{x}=L \bar{A}_{x} .
$$

And obviously the equation $P=L p$ holds.

The authors will focus their study on $p$, and the passage to $P$ is obvious.

The following equation holds: $\bar{A}_{x}=E\left(v^{T_{x}}\right)$,

where $T_{x}$ is a random variable representing the life span of a person of age $x, \bar{a}_{x}$ presents the current value of a sequence of payments of one unit each, which is paid continuously by a person as long as he is alive.

The following equation holds:

$$
\bar{a}_{x}=E\left(\frac{1-v^{T_{x}}}{\delta}\right)=\frac{1-\bar{A}_{x}}{\delta} .
$$

Equation (2) expresses a series of continuously paid insurance premiums of $p$, while the member is alive, which entitles to receive 1 from the insurance company upon his death. Equation (2) describes the balance of the whole life insurance in which the company's liabilities equal its income, 
and risk factors aren't included. This is seen following the equalities:

$$
\begin{gathered}
E\left(p \frac{1-v^{T_{x}}}{\delta}-v^{T_{x}}\right)=p \bar{a}_{x}-\bar{A}_{x} \text { and } \\
p \bar{a}_{x}-\bar{A}_{x}=0 .
\end{gathered}
$$

The insurance company has to consider a risk factor (including a profit factor).

The authors will include risk terms into the equations. If they denote $\sigma_{A}^{2}, \sigma_{A}^{2}=\operatorname{var}\left(v^{T_{x}}\right)$ and $\sigma_{a}^{2}$, $\sigma_{a}^{2}=\operatorname{var}\left(1-v^{T_{x}}\right) / \delta$, then the following equation appears: $\sigma_{a}=(1 / \delta) \sigma_{A}$.

An equation that takes under consideration the expectation of incomes and outcomes, including the risk factor, is:

$E\left(\tilde{p} \frac{1-v^{T_{x}}}{\delta}-v^{T_{x}}\right)=3\left[\operatorname{var}\left(p \frac{1-v^{T_{x}}}{\delta}-v^{T_{x}}\right)^{\frac{1}{2}}\right]$

The following holds:

$$
\begin{aligned}
& \operatorname{var}\left(\tilde{p} \frac{1-v^{T_{x}}}{\delta}-v^{T_{x}}\right)= \\
& =\left(1+\frac{\tilde{p}}{\delta}\right)^{2} v \operatorname{ar}\left(v^{T_{x}}\right)=\left(1+\frac{\tilde{p}}{\delta}\right)^{2} \sigma_{A}^{2} .
\end{aligned}
$$
If (5) into (6) and recall the equation $\sigma_{a}=\frac{1}{\delta} \sigma_{A}$,
then there follows the equation:

$$
\tilde{p}\left(\bar{a}_{x}-3 \sigma_{a}\right)=\bar{A}_{x}+3 \sigma_{A} .
$$

The meaning of equation (6) is that in case of minimum incomes, maximum outcomes should be covered. In other words, even if the company is caught into a bad financial situation, that is it receives the minimal expected amount $\bar{a}_{x}-3 \sigma_{a}$, and its payments reach the maximal expected value of $\vec{A}_{x}+3 \sigma_{A}$.

The premium $\tilde{p}$ covers a load due to the risk expected on payments and liabilities.

The authors now formulate mathematically an estimate for the variance $\sigma$ for the case of a whole life insurance, that is derived from equation (4) following the equations:
Lemma 2.1: With the above assumptions and notations, the following equation holds:

$$
\tilde{p}=\frac{p\left(1+\frac{3 \sigma_{A}}{\bar{A}_{x}}\right)}{1-\frac{3 \sigma_{a}}{\bar{a}_{x}}} .
$$

Note that the proof results from the following series of equations:

$\tilde{p}=\frac{\bar{A}_{x}+3 \sigma_{A}}{\bar{a}_{x}-3 \sigma_{a}}=\frac{\bar{A}_{x}\left(1+\frac{3 \sigma_{A}}{\bar{A}_{x}}\right)}{\bar{a}_{x}\left(1-\frac{3 \sigma_{a}}{\bar{a}_{x}}\right)}=\frac{p\left(1+\frac{3 \sigma_{A}}{\bar{A}_{x}}\right)}{1-\frac{3 \sigma_{a}}{\bar{a}_{x}}}$.

This enables the authors to derive the Macloren expansion of $\tilde{p}$ for $h=3 \sigma_{a} / \bar{a}_{x}$ as follows:

$$
\begin{aligned}
& \tilde{p}=p\left(1+\frac{3 \sigma_{A}}{\bar{A}_{x}}\right)\left(1+\frac{3 \sigma_{a}}{\bar{a}_{x}}+O\left(\frac{3 \sigma_{a}}{\bar{a}_{x}}\right)^{2}\right)= \\
& =p\left(1+\frac{3 \sigma_{A}}{\bar{A}_{x}}\right)\left(1+\frac{3 \sigma_{a}}{\bar{a}_{x}}\right)+p\left(1+\frac{3 \sigma_{A}}{\bar{A}_{x}}\right) O\left(\frac{3 \sigma_{a}}{\bar{a}_{x}}\right)^{2}= \\
& =p\left(1+\frac{3 \sigma_{A}}{\bar{A}_{x}}+\frac{3 \sigma_{a}}{\bar{a}_{x}}+\frac{3 \sigma_{A}}{\bar{A}_{x}} \frac{3 \sigma_{a}}{\bar{a}_{x}}\right)+ \\
& +p\left(1+\frac{3 \sigma_{A}}{\bar{A}_{x}}\right) O\left(\frac{3 \sigma_{a}}{\bar{a}_{x}}\right)^{2} .
\end{aligned}
$$
Since $p<1$ and $\sigma_{a}=\frac{1}{\delta} \sigma_{A}$ there follow the
equations:

$$
\begin{aligned}
& p\left(1+\frac{3 \sigma_{A}}{\bar{A}_{x}}\right) O\left(\frac{3 \sigma_{a}}{\bar{a}_{x}}\right)^{2}=p\left(\frac{3 \sigma_{a}}{\bar{a}_{x}}\right)^{2}+ \\
& p \cdot \frac{3 \sigma_{A}}{\bar{A}_{x}}\left(\frac{3 \sigma_{a}}{\bar{a}_{x}}\right)^{2}=O\left(\frac{3 \sigma_{a}}{\bar{a}_{x}}\right)^{2}+ \\
& +p \cdot \frac{3 \sigma_{A}}{\bar{A}_{x}}\left(\frac{3 \sigma_{a}}{\bar{a}_{x}}\right)^{2}=O\left(\frac{3 \sigma_{a}}{\bar{a}_{x}}\right)^{2}+ \\
& +\frac{\bar{A}_{x}}{\bar{a}_{x}} \cdot \frac{3 \sigma_{A}}{\bar{A}_{x}}\left(\frac{3 \sigma_{a}}{\bar{a}_{x}}\right)^{2}=O\left(\frac{3 \sigma_{a}}{\bar{a}_{x}}\right)^{2}+ \\
& +\frac{3 \delta \sigma_{a}}{\bar{a}_{x}}\left(\frac{3 \sigma_{a}}{\bar{a}_{x}}\right)^{2}=O\left(\frac{3 \sigma_{a}}{\bar{a}_{x}}\right)^{2}+ \\
& +\delta\left(\frac{3 \sigma_{a}}{\bar{a}_{x}}\right)^{3}=O\left(\frac{3 \sigma_{a}}{\bar{a}_{x}}\right)^{2} .
\end{aligned}
$$


Hence, the estimate of $\tilde{p}$ with $h=\frac{3 \sigma_{a}}{\bar{a}_{x}}$ is:

$$
\begin{aligned}
& \tilde{p}=p\left(1+\frac{3 \sigma_{A}}{\bar{A}_{x}}+\frac{3 \sigma_{a}}{\bar{a}_{x}}\right)+ \\
& +p \frac{3 \sigma_{A}}{\bar{A}_{x}} \frac{3 \sigma_{a}}{\bar{a}_{x}}+O\left(\frac{3 \sigma_{a}}{\bar{a}_{x}}\right)^{2} .
\end{aligned}
$$

The term $p \frac{3 \sigma_{A}}{\bar{A}_{x}} \frac{3 \sigma_{a}}{\bar{a}_{x}}$ can be simplified using the equation $\sigma_{a}=\frac{1}{\delta} \sigma_{A}$ to get:

$p \frac{3 \sigma_{A}}{\bar{A}_{x}} \frac{3 \sigma_{a}}{\bar{a}_{x}}=p \frac{3 \delta \sigma_{a}}{\bar{A}_{x}} \frac{3 \sigma_{a}}{\bar{a}_{x}}=\frac{\bar{A}_{x}}{\bar{a}_{x}} \frac{3 \delta \sigma_{a}}{\bar{A}_{x}} \frac{3 \sigma_{a}}{\bar{a}_{x}}$ that is $p \frac{3 \sigma_{A}}{\bar{A}_{x}} \frac{3 \sigma_{a}}{\bar{a}_{x}}=\delta\left(\frac{3 \sigma_{a}}{\bar{a}_{x}}\right)^{2}$.

Inserting this value to equation (8), there appears:

Lemma 2.2: With the above assumptions and notations, the following equation holds:

$$
\tilde{p}=p+3 p\left(\frac{3 \sigma_{A}}{\bar{A}_{x}}+\frac{\sigma_{a}}{\bar{a}_{x}}\right)+O\left(\frac{3 \sigma_{a}}{\bar{a}_{x}}\right)^{2},
$$

where the proof results from the series of equations:

$$
\begin{aligned}
& \tilde{p}=p\left(1+\frac{3 \sigma_{A}}{\bar{A}_{x}}+\frac{3 \sigma_{a}}{\bar{a}_{x}}\right)+O\left(\frac{3 \sigma_{a}}{\bar{a}_{x}}\right)^{2}= \\
& =p+3 p\left(\frac{\sigma_{A}}{\bar{A}_{x}}+\frac{\sigma_{a}}{\bar{a}_{x}}\right)+O\left(\frac{3 \sigma_{a}}{\bar{a}_{x}}\right)^{2} .
\end{aligned}
$$

Let us denote $\tilde{\sigma}=\frac{\sigma_{A}}{\bar{A}_{x}}+\frac{\sigma_{a}}{\bar{a}_{x}}$, namely $\tilde{\sigma}$ is the sum of the relative risk of the liabilities of the insurance company, and the relative risk of the premium payments.

Theorem 2.1: An estimate for the relative risk load

$$
\frac{\tilde{p}-p}{p} \text { is } \frac{\tilde{p}-p}{p} \approx 3 \tilde{\sigma} .
$$

\section{N-YEAR ENDOWMENT INSURANCE}

The insurance equation in the case of $n$-year endowment insurance is

$$
p \bar{a}_{x: \bar{n} \mid}=\bar{A}_{x: \bar{n} \mid},
$$

where $p$ is the net premium paid by the member to the insurance company, $\vec{A}_{x: \bar{n} \mid}$ represents the current value of one unit for a member of age $x$. The payment is given in $n$ years or in case of death.

The following holds:

$$
\bar{A}_{x: \bar{n} \mid}=E\left(v^{\min \left(T_{x}, n\right)}\right),
$$

where $\bar{a}_{x: \bar{n}}$ represents the current value of a series of payments of one unit each, paid continuously by a member of age $x$. The payments continues as long as the member is alive and up to $n$ years.

The authors have that:

$$
\bar{a}_{x: \bar{n} \mid}=E\left(\frac{1-v^{\min \left(T_{x}, n\right)}}{\delta}\right)=\frac{1-\bar{A}_{x: \bar{n} \mid} .}{\delta} .
$$

Equation (9) expresses a series of payments for $n$ years or till time of death, which ends with the member receiving one unit from the insurance company. This insurance program is called an $n$-year endowment insurance.

Like in a previous subsection, equation (9) does not include any risk factors. Again, this can be seen in the following calculation:

$$
E\left(p \frac{1-v^{\min \left(T_{x}, n\right)}}{\delta}-v^{\min \left(T_{x}, n\right)}\right)=p \bar{a}_{x: \bar{n} \mid}-\bar{A}_{x: \bar{n} \mid}=0 .
$$

To get a similar equation that will include risk factors included, let us denote:

$$
\sigma_{A}^{2}=\operatorname{var}\left(v^{\min \left(T_{x}, n\right)}\right),
$$

$$
\sigma_{a}^{2}=\operatorname{var}\left(\frac{1-v^{\min \left(T_{x}, n\right)}}{\delta}\right) \text {. }
$$


Hence, the equation with the risk factors is:

$$
\begin{aligned}
& E\left(\tilde{p} \frac{1-v^{\min \left(T_{x}, n\right)}}{\delta}-v^{\min \left(T_{x}, n\right)}\right)= \\
& =3\left[\operatorname{var}\left(\tilde{p} \frac{1-v^{\min \left(T_{x}, n\right)}}{\delta}-v^{\min \left(T_{x}, n\right)}\right)\right]^{\frac{1}{2}} .
\end{aligned}
$$

That, $\tilde{p}\left(\bar{a}_{x: \bar{n} \mid}-3 \sigma_{a}\right)=\bar{A}_{x: \bar{n} \mid}+3 \sigma_{A}$.

Lemma 3.1 results from equation (10) similarly to the way Lemma 2.1 resulted from (2.4):

Lemma 3.1: With the above insumptions and notations, the following equation holds:

$$
\tilde{p}=p \frac{1+\frac{3 \sigma_{A}}{\bar{A}_{x: \bar{n}} \mid}}{1-\frac{3 \sigma_{a}}{\bar{a}_{x: \bar{n}}}} .
$$

If we denote $\tilde{\sigma}=\frac{\sigma_{A}}{\bar{A}_{x: \bar{n} \mid}}+\frac{\sigma_{a}}{\bar{a}_{x: \bar{n} \mid}}$, then $\tilde{\sigma}$ is the sum

of the relative risk of the liabilities of the insurance company, and the relative risk of the premium payments. There appears:

Theorem 3.1: An estimate for the relative risk load $\frac{\tilde{p}-p}{p}$ is $\frac{\tilde{p}-p}{p} \approx 3 \tilde{\sigma}$.

\section{PENSION SCHEME}

The authors cannot do similar calculations as in two previous sections as will be shown next, therefore they will develop a different notion of risk estimates for the case of pension. The basic equation for the premium of pension is given in:

$$
p \bar{a}_{x: \bar{n} \mid}={ }_{n \mid} \bar{a}_{x}
$$

where $p$ is the premium paid by the member, ${ }_{n} \bar{a}_{x}$ represents current value of series of one unit payments, which are rejected for $n$ years for a member of age $x$. The payments will be paid starting from age $x+n+1$ assuming the member is alive and end at death. The following holds:
And equation (11) can be written as:

$$
{ }_{n} \bar{a}_{x}={ }_{n} p_{x} v^{n} \bar{a}_{x+n}
$$

where $\bar{a}_{x: \bar{n} \mid}$ is as in previous subsection.

Equation (11) stands for a series of payments, which are paid for $n$ years as long as the member is alive. Starting from age $x+n+1$, the member receives one unit each annually as long as he's alive. This equation does not include risk factors, this is best seen in the following calculation:

${ }_{n} q_{x} \underbrace{\left(p \bar{a}_{x: \bar{n} \mid}\right)}+{ }_{n} p_{x} \underbrace{\left(p \bar{a}_{x: \bar{n} \mid}-v^{n} \bar{a}_{x+n}\right)}=$

$=p \bar{a}_{x: \bar{n} \mid}-{ }_{n} \bar{a}_{x}$.

$A$ is the expected premiums received till age $x+n$, and $B$ is the difference between the expected premiums and the payments of the company to a member starting from age $x+n+1$. Hence, by using the corresponding probabilities, one gets the expected income for the company (namely, zero). By simple algebraic manipulations and the usage of the connection ${ }_{n} \bar{a}_{x}={ }_{n} p_{x} v^{n} \bar{a}_{x+n}$, the authors get that equation (13) is a different expression of equation (11). The variance $\sigma^{2}$ of the premiums to the company in this case is:

$\sigma^{2}={ }_{n} q_{x}\left(p \bar{a}_{x: \bar{n} \mid}\right)^{2}+{ }_{n} p_{x}\left(p \bar{a}_{x: \bar{n} \mid}-v^{n} \bar{a}_{x+n}\right)^{2}$

Using equation (14), the equation ${ }_{n} p_{x}+{ }_{n} q_{x}=1$ and the equation: ${ }_{n} \bar{a}_{x} /{ }_{n} p_{x}=v^{n} \bar{a}_{x+n}$, one rearranges (14) as follows:

$$
\begin{aligned}
& \sigma^{2}={ }_{n} q_{x}\left(p \bar{a}_{x: \bar{n} \mid}\right)^{2}+{ }_{n} p_{x}\left(p \bar{a}_{x: \bar{n} \mid}-v^{n} \bar{a}_{x+n}\right)^{2}= \\
& =\frac{1}{{ }_{n} p_{x}^{2}}\left({ }_{n} q_{x n} p_{x}^{2}\left({ }_{n} \bar{a}_{x}\right)^{2}+{ }_{n} p_{x}^{3}\left({ }_{n} \bar{a}_{x}-v^{n} \bar{a}_{x+n}\right)^{2}\right)= \\
& =\frac{1}{{ }_{n} p_{x}^{2}}\left({ }_{n} q_{x n} p_{x}^{2}\left({ }_{n \mid} \bar{a}_{x}\right)^{2}+{ }_{n} p_{x}^{3}\left({ }_{n \mid} \bar{a}_{x}-\frac{{ }_{n} \bar{a}_{x}}{{ }_{n} p_{x}}\right)^{2}\right)= \\
& =\frac{1}{{ }_{n} p_{x}^{2}}\left({ }_{n} q_{x n} p_{x}^{2}\left({ }_{n} \bar{a}_{x}\right)^{2}+{ }_{n} p_{x}^{3}\left(\left({ }_{n \mid} \bar{a}_{x}\right)^{2}\left(1-\frac{1}{{ }_{n} p_{x}}\right)^{2}\right)\right)=
\end{aligned}
$$$$
=\frac{1}{{ }_{n} p_{x}^{2}}\left({ }_{n} q_{x n} p_{x}^{2}\left({ }_{n \mid} \bar{a}_{x}\right)^{2}+{ }_{n} p_{x}^{3}\left(\left({ }_{n} \bar{a}_{x}\right)^{2}\left(-\frac{{ }_{n} q_{x}}{{ }_{n} p_{x}}\right)^{2}\right)\right)=
$$$$
=\frac{1}{{ }_{n} p_{x}^{2}}\left({ }_{n} q_{x n} p_{x}\left({ }_{n \mid} \bar{a}_{x}\right)^{2}\left({ }_{n} p_{x}+{ }_{n} q_{x}\right)\right)=\frac{{ }_{n} q_{x}\left({ }_{n} \bar{a}_{x}\right)^{2}}{{ }_{n} p_{x}} \text {. }
$$ 
Since ${ }_{n !} \bar{a}_{x}={ }_{n} p_{x} v^{n} \bar{a}_{x+n}$, one derives the equation $\sigma=v^{n} \bar{a}_{x+n} \sqrt{{ }_{n} p_{x n} q_{x}}$. Therefore, equation (11) taking into account the risk factor becomes:

$$
\tilde{p} \bar{a}_{x: \bar{n}}-{ }_{n} p_{x} v^{n} \bar{a}_{x+n}=3 \sigma
$$

which can also be written as:

$$
\tilde{p} \bar{a}_{x: \bar{n} \mid}-{ }_{n} p_{x} v^{n} \bar{a}_{x+n}=3 v^{n} \bar{a}_{x+n} \sqrt{{ }_{n} p_{x n} q_{x}} .
$$

Subtracting equation (12) from equation (15), the following equation appears.

Lemma 4.1: With the above assumptions and notations, the following equation holds:

$$
\tilde{p} \bar{a}_{x: \bar{n} \mid}-p \bar{a}_{x: \bar{n} \mid}=3 v^{n} \bar{a}_{x+n} \sqrt{{ }_{n} p_{x n} q_{x}},
$$

by dividing the equation $\tilde{p} \bar{a}_{x: \bar{n} \mid}-p \bar{a}_{x: \bar{n} \mid}=3 v^{n} \bar{a}_{x+n} \sqrt{{ }_{n} p_{x n} q_{x}}$ by the equation $p \bar{a}_{x: \bar{n} \mid}={ }_{n} p_{x} v \bar{a}_{x+n}$, the following equation appears:

$$
\frac{\tilde{p}-p}{p}=3 \sqrt{\frac{{ }_{n} q_{x}}{{ }_{n} p_{x}}} .
$$

Finally, there appears the following equation:

$$
\sqrt{\frac{{ }_{n} q_{x}}{{ }_{n}}}=\frac{\sigma\left(v^{n} \bar{a}_{\max \left(T_{x, n}\right)-n}\right)}{E\left(v^{n} \bar{a}_{\max \left(T_{x, n}\right)-n}\right)},
$$

with the expected payments of pension $E\left(v^{n} \bar{a}_{\max \left(T_{x, n}\right)-n}\right)$ and its corresponding variance $\operatorname{Var}\left(v^{n} \bar{a}_{\max \left(T_{x, n}\right)-n}\right)$, one verifies that:

$$
\begin{aligned}
& \left(v^{n} \bar{a}_{\max \left(T_{x, n}\right)-n}\right)=v^{n} E\left(\bar{a}_{\max \left(T_{x, n}\right)-n}\right)= \\
& =v^{n}\left({ }_{n} q_{x} \cdot 0+{ }_{n} p_{x} \bar{a}_{x+n}\right)=v^{n}{ }_{n} p_{x} \bar{a}_{x+n}
\end{aligned}
$$

and

$$
\begin{aligned}
& V=\operatorname{Var}\left(v^{n} \bar{a}_{\max \left(T_{x, n}\right)-n}\right)={ }_{n} q_{x}\left(0-{ }_{n} p_{x} v^{n} \bar{a}_{x+n}\right)^{2}+ \\
& +{ }_{n} p_{x}\left(v^{n} \bar{a}_{x+n}-v^{n}{ }_{n} p_{x} \bar{a}_{x+n}\right)^{2}= \\
& ={ }_{n} q_{x n} p_{x}^{2}\left(v^{n} \bar{a}_{x+n}\right)^{2}+{ }_{n} p_{x}\left(v^{n} \bar{a}_{x+n}\right)^{2}\left(1-{ }_{n} p_{x}\right)^{2}= \\
& ={ }_{n} q_{x n} p_{x}\left(v^{n} \bar{a}_{x+n}\right)^{2}\left({ }_{n} p_{x}+{ }_{n} q_{x}\right)={ }_{n} q_{x n} p_{x}\left(v^{n} \bar{a}_{x+n}\right)^{2},
\end{aligned}
$$

therefore,

$$
\frac{\sigma\left(v^{n} \bar{a}_{\max \left(T_{x, n}\right)-n}\right)}{E\left(v^{n} \bar{a}_{\max \left(T_{x, n}\right)-n}\right)}=\frac{v^{n} \bar{a}_{x+n} \sqrt{{ }_{n} q_{x n} p_{x}}}{v^{n}{ }_{n} p_{x} \bar{a}_{x+n}},
$$

or

$$
\frac{v^{n} \bar{a}_{x+n} \sqrt{{ }_{n} q_{x n} p_{x}}}{v^{n}{ }_{n} p_{x} \bar{a}_{x+n}}=\sqrt{\frac{{ }_{n} q_{x}}{{ }_{n} p_{x}}},
$$

which implies the validity of equation (18).

If we denote $\tilde{\sigma}=\frac{\sigma_{A}}{\bar{A}_{x}}+\frac{\sigma_{a}}{\bar{a}_{x}}$, then $\tilde{\sigma}$ is the sum of the relative risk of the liabilities of the insurance company, and the relative risk of the premium payments, and there appears:

Theorem 4.1: An estimate for the relative risk load

$$
\frac{\tilde{p}-p}{p} \text { is } \frac{\tilde{p}-p}{p} \approx 3 \tilde{\sigma} \text {. }
$$

In terms of equations (18) and (17), the relative risk load is the ratio of the expected deviation of premiums to the expected pension payments. That is,

$$
\frac{\tilde{p}-p}{p}=3 \frac{\sigma\left(v^{n} \bar{a}_{\max \left(T_{x, n}\right)-n}\right)}{E\left(v^{n} \bar{a}_{\max \left(T_{x, n}\right)-n}\right)} .
$$

The standard deviation $\sigma\left(v^{n} \bar{a}_{\max \left(T_{x, n}\right)-n}\right)$ is independent of the paid premiums, as pension is paid only if all premiums are successfully completed, and the relative risk load is 3 times the relative deviation of the pension ${ }_{n \mid} \bar{a}_{x}=v^{n} \bar{a}_{\max \left(T_{x, n}\right)-n}$.

\section{EXAMPLE}

The authors study an example of a relative risk load for a person of age 35 insured in a whole life insurance of $100,000 \$$, assuming the insurance is based upon the ELT-12 life table with a yearly interest of $3 \%$.

The authors study the relative risk load for a single person, for a group of 1,000 insured persons and for a group of 10,000 insured persons. 
The various cases help to evaluate the effect on the relative risk load of the size of the insured group with similar policies, thus emphasizing the importance of large groups of insured person to reduce the risk of an insurance plan.

Using the given life table and interest, the authors evaluated the following symbols:

$$
\begin{gathered}
\bar{a}_{35}=21.5335, \bar{A}_{35}=0.3636, \\
\sigma_{a}=4.654127, \sigma_{A}=0.013748,
\end{gathered}
$$

from which the classical risk factors are derived as:

$$
\frac{\sigma_{a}}{\bar{a}_{35}}=0.21613, \frac{\sigma_{A}}{\bar{A}_{35}}=0.37811
$$

therefore, the deviation $\tilde{\sigma}$ is evaluated as

$$
\tilde{\sigma}=\frac{\sigma_{a}}{\bar{a}_{35}}+\frac{\sigma_{A}}{\bar{A}_{35}}=0.59424
$$

The relative risk load for an insured person as a single person is:

$$
\frac{\delta}{p}=\frac{\tilde{p}-p}{p}=3 \tilde{\sigma}=3 \cdot 0.59424=178.272 \%,
$$

the relative risk load is reduced in a group of 1,000 persons to: $\frac{\delta}{p}=\frac{\tilde{p}-p}{p}=3 \frac{\tilde{\sigma}}{\sqrt{1,000}}=\frac{178.272 \%}{\sqrt{1,000}}=5.637 \%$, and the relative risk load is farther reduced in a group of 10,000 persons to:

$\frac{\delta}{p}=\frac{\tilde{p}-p}{p}=3 \frac{\tilde{\sigma}}{\sqrt{10,000}}=\frac{178.272 \%}{\sqrt{10,000}}=1.783 \%$.

The net premium for the insurance is

$$
P=100,000 \frac{\bar{A}_{35}}{\bar{a}_{35}}=100,000 \frac{0.3636}{21.5335}=1,689 \$ .
$$

The gross premium for a single person for the insurance includding a risk load is:

as a single person

$\tilde{P}=P(1+\delta)=1,689 \cdot 2.78272=4,700 \$$,

in a group of 1,000 persons

$\tilde{P}=P(1+\delta)=1,689 \cdot 1.05637=1.784 \$$,

in a group of 10,000 persons

$\tilde{P}=P(1+\delta)=1,689 \cdot 1.01783=1,719 \$$.

\section{CONCLUSION}

In this article, the authors investigate financial portfolios for groups of members under similar risk factors. The risks are subject to classical type statistics, that is, the larger the groups, the smaller the risk. Obviously, a reduced risk provide an emergence of benefit to the management of the portfolio. Larger groups occur when several groups which share similar portfolios join under one management whether by buying each other, by joining administrations, or by any other form of joint operation. Groups with similar financial risks that may consider a joint operation occur as mortgage loans, pension funds with similar schemes, mutual funds, whole life and short-term insurance plans.

The risk may be evaluated by statistical methods or otherwise, and being assumed to decrease with the increase in the portfolio in case of biying a portfolio, the buyer obviously receives all the emerging benefit and in case of joining management, the emerging benefit will be split between the two groups.

The results of this article may be applied in case of mergence of various portfolios of a similar type of risk. This occurs in the various cases that were mentioned above as the mortgage loans, the pension funds, the mutual funds, the whole life and the short-term insurance plans, and elementary insurance plans as property insurance plans and car insurance plans and health insurance plans. It is the basis for reinsurance business.

The authors study the emerging benefit in some of the cases of mergence of portfolios that are subject to similar risks. The authors proceed to discuss the splitting of the emerging benefit between the various merging portfolios. 
In this article, the authors discuss the models of financial portfolios that carry some type of management fee and some risk liabilities. The management fee is a payment to the company that manages the portfolio. In this case, managing larger groups results in a profit to the company over the increase in the management due to the enlarged group.

The authors emphasize the discussion to the management of the risk liabilities. When risk liabilities are extenal, like in some insurance plans, the company carries responsibility for the risk, it is like the case of the management fee to the company.

When the risk liabilities are internal, like in some pension schemes, the benefit from the reduced risk in case of a mergence process belongs to the members of the scheme.

One should consider the parties entitled to the benefit that emerges in the process, of expanding via bidding for another portfolio, or merging with another portfolio.

The evaluation of the emerging benefit of the extended portfolio is crucial in the process of extending the portfolio whether by mergence, by buying, or by joining the management of the two portfolios.

\section{REFERENCES}

1. Borch, K. (1974). The Mathematical Theory of Insurance. D. C. Heath \& Comp.

2. Brower, N. L., Gerber, H. U., Hickman, J. C., Jones, D. A., \& Nesbitt, C. J. (1997). Actuarial Mathematics. The Society of Actuaries.

3. Conitzer, V., \& Sandholm, T. (2004). Computing Shapley values, manipulating value division schemes, and checking core membership in multi-issue domains (19th nat. conf. Art.). AAAI, 219-225. Retrieved from https://users.cs.duke.edu/ conitzer/ shapleyAAAI04.pdf

4. Date, P., Mamon, R., \& Wang, I. C. (2007). Valuation of cash flows under random rates of interest: A linear algebraic approach. Insurance Mathematics and Economics, 41(1), 84-95. http:// dx.doi.org/10.1016/j.insmatheco.2006.10.001

5. Fishburn, P. C. (1994). Utility and Subjective Probability. Handbook of Game Theory with Economic Applications, 2, 1397-1435. https://doi.org/10.1016/S15740005(05)80071-2

6. Gerber, H. U. (1997). Life Insurance Mathematics. Springer-Verlag Berlin Heidelberg. Retrieved from https://www.springer.com/us/

book/9783540622420

7. Kaas, R., Goovaerts, M., Dhaene, J., \& Denuit, M. (2008). Modern Actuarial Risk Theory. Using R. Springer-Verlag Berlin Heidelberg. Retrieved from https://www.springer.com/us/book/9783540709923

8. Kahneman, D., \& Tversky, A. (1979). Prospect Theory: An Analysis of Decision Under Risk. Econometrica, 47(2), 263-291. http://dx.doi.org/10.2307/1914185

9. Knaster, B., Kuratowski C., \& Mazurkiewicz, C. (1929). Ein Beweis de Fixpunktsatzes fuer n-dimensionale Simplexe. Fundamenta Mathematicae, 14(1), 132-137. Retrieved from https:// eudml.org/doc/212127

10. Lemaire, J. (1979). Reinsurance as a Cooperative Game. Applied Game Theory, 254-269. Retrieved from https://link.springer.com/chapter/10.1007/978-3-662-41501-6_16

11. McCutcheon, J. J., Scott, W. F. (1991). An Introduction to the Mathematics of Finance. Butterworth-Heinemann, Oxford.

12. Mualem, E., \& Zaks, A. (2017). Joining insured groups: How to split the emerging profit. Insurance Markets and Companies, 8(1),
29-33. http://dx.doi.org/10.21511/ ins.08(1).2017.03

13. Mualem, E., \& Zaks, A. (2018). Fair Split of Profit generated by $n$ Parties. Insurance Markets and Companies, 9(1), 6-9. http://dx.doi. org/10.21511/ins.09(1).2018.01

14. Neill, A. (1989). Life contingencies. Heinemann Pro. Pub. Oxford.

15. Shapley, L. S. (1929). Cores of Convex Games. International Journal of Game Theory, 1, 11-26. Retrieved from https://link.springer.com/ article/10.1007\%2FBF01753431

16. Zaks, A. (2001). Annuities under random rates of interest. Mathematics and Economics, 28, 1-11. Retrieved from https://pdfs. semanticscholar.org/40bb/986fa87b 5509f9610aa235feff9adb46a8db.pdf

17. Zaks, A. (2009). Annuities under changes in Life Tables and changes in the Interest. Pravartak J. Ins. \& Risk Man. 8, 47-52. Retrieved from https://papers.ssrn.com/sol3/papers. cfm?abstract_id $=1412223$

18. Zaks, A. (2010). Annuities Under Random Rates of Interest II. Pravartak J. Ins. \& Risk Man., 5(2), 59-65. Retrieved from https:// papers.ssrn.com/sol3/papers. cfm?abstract_id $=1397136$ 